Article

\title{
Degree of Branching in Hyperbranched Poly(glycerol-co-diacid)s Synthesized in Toluene
}

\section{Victor T. Wyatt * and Gary D. Strahan}

United States Department of Agriculture, Agricultural Research Service, Eastern Regional Research Center, Wyndmoor, PA 19038, USA; E-Mail: gary.strahan@ars.usda.gov

* Author to whom correspondence should be addressed; E-Mail: victor.wyatt@ars.usda.gov; Tel.: +1-215-233-6674; Fax: +1-215-233-6795.

Received: 1 January 2012; in revised form: 28 January 2012 / Accepted: 31 January 2012 / Published: 6 February 2012

\begin{abstract}
H}$ NMR and ${ }^{13} \mathrm{C}$ NMR spectrometry (1-dimensional and 2-dimensional) have been used to assign chemical resonances and determine the degrees of branching for polyesters synthesized by the Lewis acid (dibutyltin(IV)oxide)-catalyzed polycondensation of glycerol with either succinic acid (n (aliphatic chain length) $=2$ ), glutaric acid $(n=3)$ or azelaic acid $(n=7)$ in quasi-melt solutions with toluene. When $1: 1$ and 2:1 (diacid:glycerol) molar ratios were used, it was found that the glutaric acid-derived polymers gave the highest degree of polymer branching (31.2\%, 85.6\%, respectively) after the $24 \mathrm{~h}$ reaction period followed by the succinic acid-derived polymers (39.4\%, 41.9\%, respectively) and the azelaic acid-derived polymers (9.9\%, 13.9\%, respectively). Reactions performed at reflux for $24 \mathrm{~h}$ resulted in a $70.8 \%$ and $56.7 \%$ decrease in degree of branching for succinic acid and glutaric acid-derived polyesters, respectively. There is no indication that degree of branching is significantly affected by the presence or absence of solvent according to the results obtained in this research.
\end{abstract}

Keywords: diacids; hyper-branching; glycerol; polymers

\section{Introduction}

Degree of branching (D.B.\%) has been well studied in systems that involve the direct condensation reaction between difunctional $A_{2}$ monomers, such as diacids, and trifunctional $B_{3}$ monomers, such as 
glycerol, in the presence of lipase and chemical catalysts to form hyperbranched polymers [1-7]. Understanding the relationship between D.B\% and the physical and chemical properties of hyperbranched polymers is necessary to identify potential applications. Degree of branching is a structural property that is determined by the concentration of linear (L), terminal (T) and dendritic (D) units within the polymer matrix. Dendritic (treelike) macromolecules, dendrimers and hypebranched polymers, have received much attention in recent years because their highly branched structures and large number of terminal functional groups are expected to produce materials with unique properties when compared to their linear analogues [8-10]. Dendrimers are produced by tedious step-wise processes that avoid gelation and lead to perfectly branched (100\%) products [2,8]. There are no unreacted functional groups or linear units along the branched chains of dendrimers. Conversely, hyperbranched polymers are prepared by one-pot processes whereby difunctional $\left(\mathrm{A}_{2}\right)$ and trifunctional $\left(B_{3}\right)$ monomers are reacted at random yielding products with $15-90 \%$ branching $[6,11-14]$.

The higher concentration of unreacted functional groups along the branched chains make hyperbranched polymers more susceptible to the formation of network (crosslinked) polymers. Flory was the first to describe the critical gel-point for hyperbranched polymers produced in $A_{2}+B_{3}$ systems [15]. Increasing the degree of polymerization increases the number of free functional groups along the branched chain resulting in an increased probability of gel formation. Therefore, several methods have been employed to avoid gelation as degrees of branching increase in direct $A_{2}+B_{3}$ condensation reactions by performing the reactions in dilute solutions $[1,4,5]$ ) or by reacting them in the absence of solvent while monitoring the viscosity of the system [2]. Others have shown that gelation, branching, and molecular weight can be controlled by lipase-catalyzed, bulk polycondensations [3].

In previous studies, degrees of branching had been increased and crosslinking avoided when protocols for the synthesis of hyperbranched polymers were performed in dilute solutions [12]. We have synthesized hyperbranched poly(diacid-glycerol)s in nonpolar media [16] and in aprotic polar media [17]. In dilute solutions, decreased interactions between propagating chains decrease crosslinking and increase the degree of polymerization when compared to neat polymerizations. This work examines some of the conditions by which branching can be increased while avoiding gelation in a dilute solution when using free glycerol as a reactant to form polyglycerols. Glycerol-based polymers are expected to render new surgical materials that are useful in orthopedic and ophthalmic applications, reconstructive surgery and as drug delivery agents [18-20]. Such biocompatible polymers also may also be of use as cosmetics, food additives, surfactants, lubricants, and azeotrophic phase separators [21]. Toward this goal, a series of diacids were reacted with glycerol in the presence of toluene. NMR spectroscopy was used to determine the effect of toluene on gelation and the degree of branching.

\section{Materials and Methods}

\subsection{Materials}

Succinic acid (99+\%), azelaic acid (98\%), glutaric acid (99\%), reagent grade glycerol (99.9\%), anhydrous tetrahydrofuran (99.9\%), and HPLC grade toluene (99.8\%) were purchased from Sigma Aldrich (St. Louis, MO, USA). Deuterated NMR solvent, dimethylsulfoxide- $\mathrm{d}_{6}$, was ordered from Cambridge Isotope Laboratories (Andover, MA, USA). HPLC grades of acetone, chloroform, and 
methanol were purchased from J.T. Baker (Phillipsburg, NJ, USA). Sodium carbonate (anhydrous, granular) was obtained from Mallinckrodt-Baker, Inc. (Paris, KY, USA).

\subsection{Polymerizations in Toluene}

Poly(diacid-glycerol)s were synthesized as previously described [16]. A 2:1 molar ratio of diacid $(0.02 \mathrm{~mol})$ and glycerol $(0.0105 \mathrm{~mol})$ or a $1: 1$ molar ratio of diacid $(0.015 \mathrm{~mol})$ and glycerol $(0.0155 \mathrm{~mol})$ were reacted in the presence of $0.15 \% \mathrm{w} / \mathrm{w}$ (catalyst/reactants) dibutyltin(IV)oxide in a single-neck $50 \mathrm{~mL}$ round bottom flask with $28 \mathrm{~mL}$ of toluene. Experiments were performed at oil bath temperatures of $110^{\circ} \mathrm{C}, 135^{\circ} \mathrm{C}$ and $155^{\circ} \mathrm{C}$ for $10 \mathrm{~h}, 24 \mathrm{~h}$, or $72 \mathrm{~h}$. A reflux condenser was connected to the top of a Dean-Stark apparatus used to remove water over the course of the esterifications. Following reaction, most of the solvent was decanted from the crude reaction products and the rest was removed by rotary evaporation. The diacids were extracted from their polymers by three centrifugations in water at 4,000 rpm for $10 \mathrm{~min}$ at $21^{\circ} \mathrm{C}$. The water was decanted off, the polymer was dissolved in THF, and then allowed to stand overnight in sodium carbonate. After vacuum filtration, the THF was removed by rotary evaporation.

\subsection{Neat Polymerizations}

Poly(diacid-glycerol)s were synthesized as previously described [16]. The diacid (0.20 mol), glycerol $(0.105 \mathrm{~mol})$ and $0.15 \% \mathrm{w} / \mathrm{w}$ dibutyltin(IV)oxide (catalyst/reactants), were placed in a $100 \mathrm{~mL}$ two-neck round bottom flask and heated to $60^{\circ} \mathrm{C}$ under reduced pressure ( $\left.\sim 50 \mathrm{~Pa}\right)$ for one hour. The reaction mixture subsequently was allowed to react for $2 \mathrm{~h}$ at $100{ }^{\circ} \mathrm{C}$, then $2 \mathrm{~h}$ at $120^{\circ} \mathrm{C}$, and finally at $150{ }^{\circ} \mathrm{C}$ under reduced pressure ( 150 Pa), for either $10 \mathrm{~h}$ or $24 \mathrm{~h}$.

\subsection{Nuclear Magnetic Resonance (NMR)}

Solution-state NMR spectra were recorded at 9.4 Tesla on a Varian INOVA NMR Spectrometer at $27{ }^{\circ} \mathrm{C}$, using a $5 \mathrm{~mm}$ indirect-detect probe with Z-axis pulsed field gradient. All samples were dissolved in $d_{6}$-DMSO with TSP added for referencing. The $1 \mathrm{D}$ proton $\left({ }^{1} \mathrm{H}\right)$ spectra $(400 \mathrm{MHz})$ were acquired with a spectral width of $6,000 \mathrm{~Hz}$, a 90-degree pulse angle and a 2.5 s relaxation delay, and were referenced to the TSP peak. The ${ }^{13} \mathrm{C}$ spectra $(100 \mathrm{MHz})$ were acquired with a spectral width of $25-30 \mathrm{kHz}$, a pulse angle of $45^{\circ}$, a relaxation delay of $20 \mathrm{~s}, 128 \mathrm{~K}$ data points, and 6,000-18,000 transients. These spectra were referenced to the DMSO methyl carbon (39.5 ppm). To allow for accurate integration of ${ }^{13} \mathrm{C}$ signals, these $1 \mathrm{D}-{ }^{13} \mathrm{C}$ spectra were recorded with proton decoupling only during the acquisition periods ("inverse-gating”), to avoid non-uniform enhancement of carbon signals from proton NOE effects. No attempt was made to measure the $\mathrm{T} 1$ relaxation of the ${ }^{13} \mathrm{C}$ resonances, however, the relaxation delay of $20 \mathrm{~s}$, combined with the $45^{\circ}$ excitation pulse, helped to minimize any distortions due to incomplete relaxation.

Resonance assignments utilized the DEPT (Distortionless Enhancement Polarization Transfer) spectra, as well as 2D-NMR. A DEPT experiment was run using the standard $135^{\circ}$ flip angle to distinguish amongst $\mathrm{CH}, \mathrm{CH}_{2}$ and $\mathrm{CH}_{3}{ }^{13} \mathrm{C}$ resonances. The 2D-NMR studies included gradient enhanced versions of COSY (Correlation Spectroscopy), HSQC (Heteronuclear Single Quantum 
Correlation) and HMBC (Heteronuclear Multiple Bond Correlation) (8 and $16 \mathrm{~Hz}$ coupling) experiments, and non-gradient versions of the HMQC (Heteronuclear Multiple Quantum Correlation) and TOCSY (Total Correlation Spectroscopy) (80 ms mixing time) experiments. The proton homonuclear COSY and TOCSY (80 ms mixing time) 2D experiments were recorded with spectral widths of 6 or $8 \mathrm{kHz}$ in both dimensions, using 4,096 points in the directly-detected dimension, and 512 increments in the second dimension; 16 transients per fid were collected with a $2 \mathrm{~s}$ delay between scans.

The ${ }^{1} \mathrm{H}-{ }^{13} \mathrm{C}$ heteronuclear experiments, HSQC, HMQC and HMBC (8 Hz and $16 \mathrm{~Hz}$ coupling), were recorded using using 4,096 data points and spectral widths of $6 \mathrm{kHz}$ in the proton dimension (directly-detected). For the carbon (indirectly-detected) dimension, 512 increments were acquired with a spectral width of 25 or $30 \mathrm{kHz}$, a 2 s delay between scans, and 32 or 64 transients per fid.

${ }^{1} \mathrm{H}$ NMR of white powder after vacuum filtration of azelaic acid-glycerol polymer (DMSO- $\mathrm{d}_{6}$, $200 \mathrm{MHz} ; \mathrm{R}=\mathrm{H}$ or Ester)): $\delta 1.25$ (m, $\left.-\mathrm{CH}_{2}-\mathrm{C}_{2}-\mathrm{CH}_{2}-\right), \delta 1.48\left(\mathrm{~m},-\mathrm{CH}_{2}-\right), \delta 2.19\left(\mathrm{t},-\mathrm{C}_{2}-\mathrm{COOH}\right)$.

\section{Results and Discussion}

\subsection{NMR Assignments and Integrations}

The synthesis and analytical charaterization of these polymers have been previously reported [16]. Therefore, information regarding yields, solubilities, and molecular weights is available. Similar NMR peak assignments have also been published by ourselves and others [2,3,17].

Figure 1. Subsititution patterns for the glycerol backbone after reaction with one or more diacids. Carbons are labeled for NMR spectral assignments.
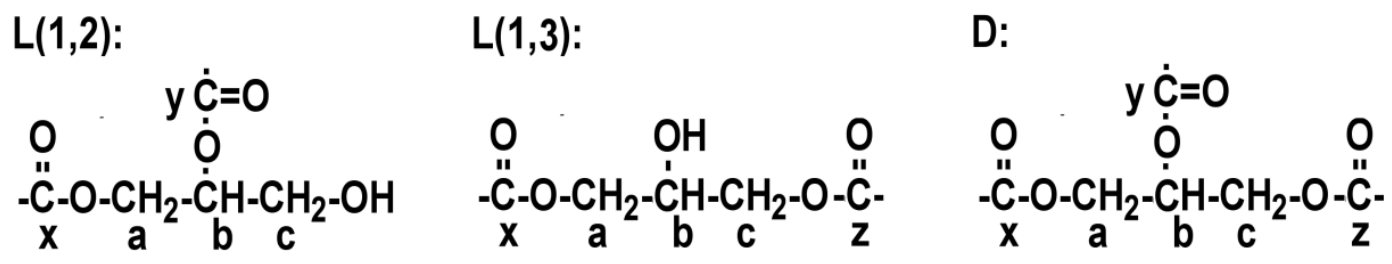

$T(1,3):$

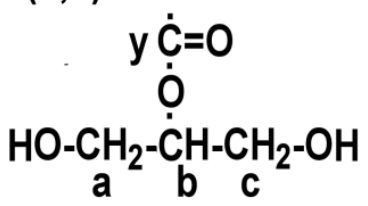

TG:

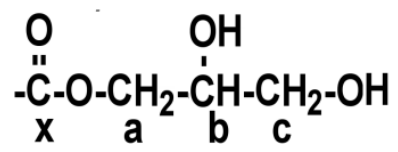

In this study, ${ }^{1} \mathrm{H}$ and ${ }^{13} \mathrm{C}$ NMR have been employed to determine how many glycerol units have been esterified at the secondary alcohol to form branched polymers. The glycerol backbone of each hyperbranched structure can be composed of trisubstitued dendritic (D), disubstituted linear (L), and terminal (T) units as shown in Figure 1. Determining the degree of branching (D.B.\%) by NMR relies upon the ability to distinguish between these units and the ability to thereby assign the carbons at the branch points and the terminal units as shown in Figure 1 . The ${ }^{1} \mathrm{H}$ and ${ }^{13} \mathrm{C}$ NMR resonance peaks associated with these units can then be integrated and used to calculate the degree of branching according to Equation (1) [2,3,6,13,17,22-24]. This equation is more appropriate for $A_{2}+B_{3}$ 
hyperbranched polymer systems because, unlike the equation introduced by Hawker [25] and modified by Kim [26], it is valid for both low and high molecular weight hyperbranched polymers and oligomers. It is also more reliable when monomer feed compositions are varied [6] such as in the experiments reported here.

$$
\mathrm{DB} \%=\frac{2 \mathrm{D}}{2 \mathrm{D}+\left(\mathrm{L}_{1,2}+\mathrm{L}_{1,3}\right)} \times 100
$$

In this work, we have performed DEPT and 2D NMR to assign the NMR resonances using through-bond connectivity, rather than depend on chemical shift analyses, which are less reliable and can be misleading. The proton resonances were thus assigned, and compared favorably with published data [2,3]. A review of the literature reveals that the exact assignment of the carbon resonances (especially the central carbon in the glycerol subunit in the $T_{G}$ and $L(1,2)$ branching structures) may be dependent upon the chemical composition of the branched chains. The HMBC experiment was of particular importance, as it enabled the definitive determination of the chemical connectivity from the carbonyl carbons of the diacid to the glycerol carbons, through the ester linkage. The resulting assignments are largely consistent with those of Kulshrestha et al. [3] but differed from those of Stumbe and Bruchmann [2], presumably because of slight differences in chemical environments in the different polymers. Whereas the ${ }^{13} \mathrm{C}$ spectra generally produced sharper, and more resolvable peaks, the ${ }^{1} \mathrm{H}$ resonances were broader and required careful baseline subtraction to obtain useful integrations. The ${ }^{13} \mathrm{C}$ integrations and degree of branching analysis should be therefore considered more accurate and reliable, but comparison with the ${ }^{1} \mathrm{H}$ values is useful for confirmation of the analyses.

Figure 2. The 2D-HMQC of the succinic acid:glycerol polymer (1:1 molar ratio, $\left.110{ }^{\circ} \mathrm{C}\right) .{ }^{\mathrm{a}}$

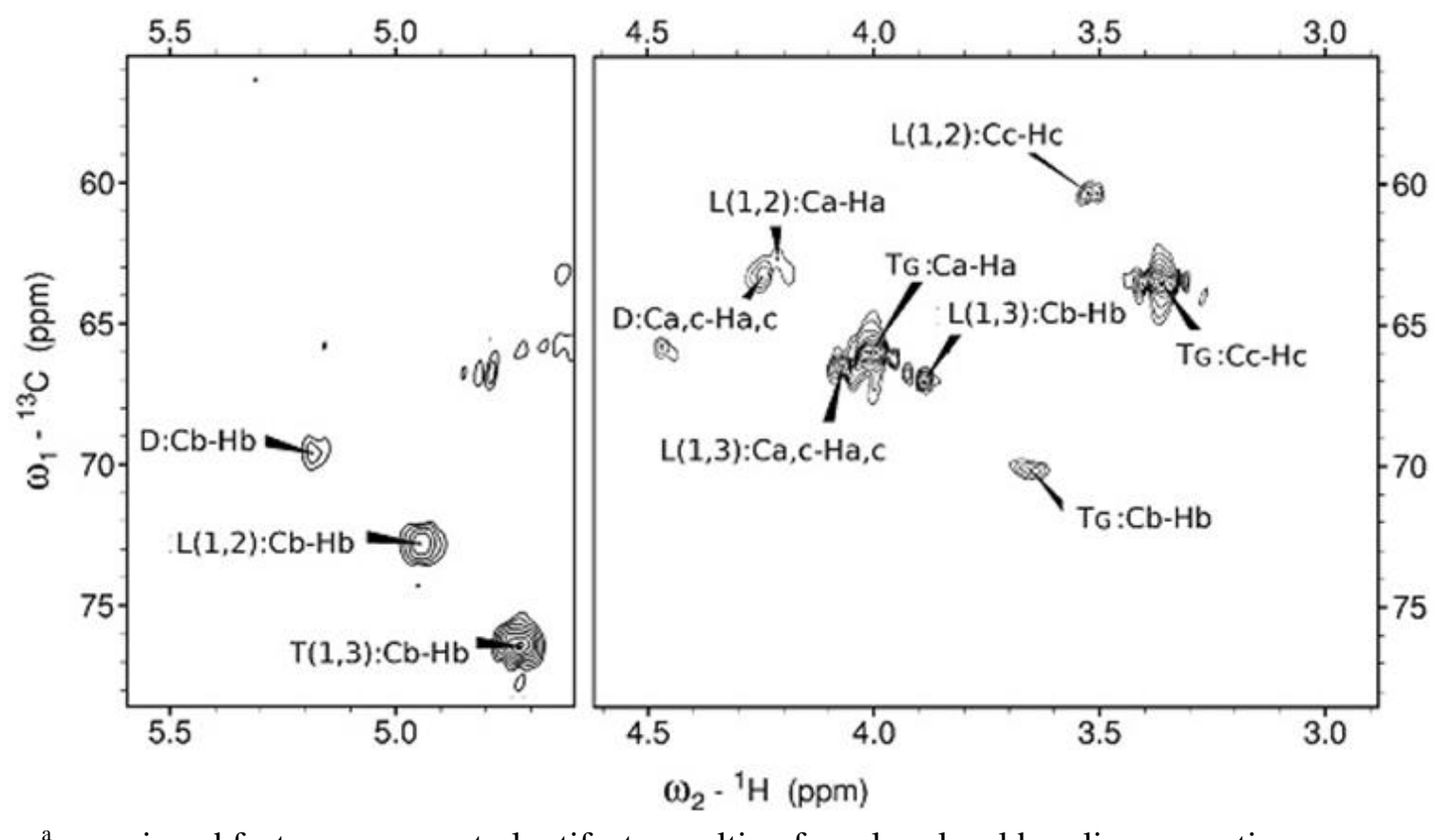

${ }^{a}$ unassigned features are spectral artifacts resulting from low-level baseline correction errors. 
The ${ }^{1} \mathrm{H}$ and ${ }^{13} \mathrm{C}$ assignments for the glycerol region are depicted in the HMQC spectra (Figure 2). A complete listing of the NMR assignments for the glycerol region is provided as supplementary material and a condensed version of the average resonance assignments appears in Table 1. Representative $1 \mathrm{D}-{ }^{13} \mathrm{C}$ spectra of our oligomers are shown in Figure 3. Some of the resonances listed in Table 1 were assigned from 2D analyses that may not be visible on the scale depicted in the 1D spectra. As expected the resonance frequencies of the glycerol carbon atoms (positions a-c. Figure 1) are independent of the $\mathrm{A}_{2}: \mathrm{B}_{3}$ monomer ratio or the reaction temperature, but are more dependent upon the substitution pattern of the glycerol unit (Table 1). Although the chemical shift values of a, b and c of the different subunits very similar for each of the diacid derivatives, slight variations arise from the different diacids that have be incorporated.

Figure 3. The glycerol region of representative, inverse-gated ${ }^{13} \mathrm{C}$ NMR spectra of the indicated diacids copolymerized with glycerol.

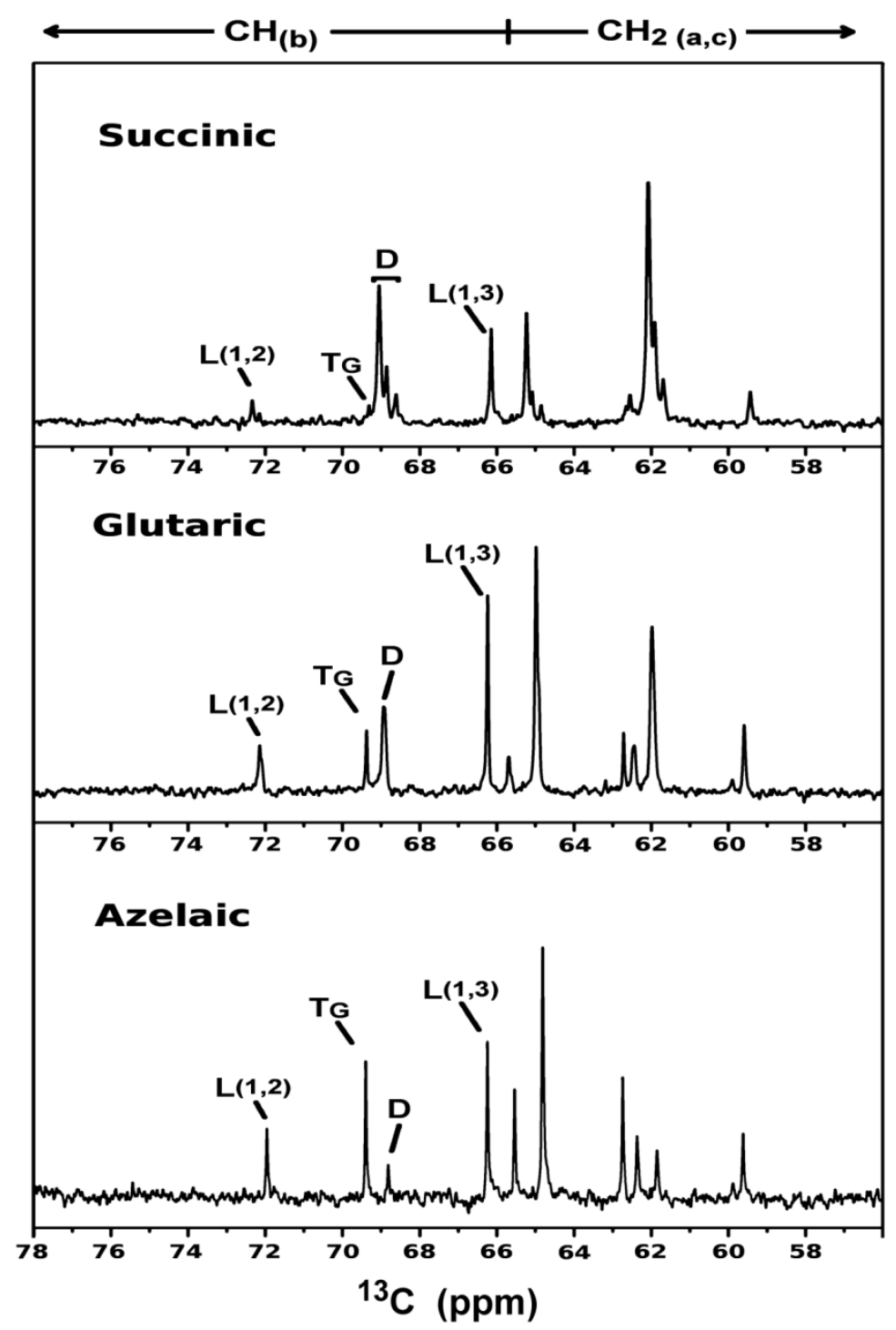


Table 1. Averaged NMR resonance assignments (with standard deviations) for diacid acid-glycerol polymer branching patterns as shown in Figure 1.

\begin{tabular}{|c|c|c|c|c|c|c|}
\hline \multirow{2}{*}{$\begin{array}{c}\text { Atom position in } \\
\text { branching pattern } \\
\text { PPM }\end{array}$} & \multicolumn{2}{|c|}{ Glutaric Acid:Glycerol } & \multicolumn{2}{|c|}{ Succinic Acid:Glycerol } & \multicolumn{2}{|c|}{ Azelaic Acid:Glycerol } \\
\hline & ${ }^{1} \mathbf{H}$ & ${ }^{13} \mathrm{C}$ & ${ }^{1} \mathbf{H}$ & ${ }^{13} \mathrm{C}$ & ${ }^{1} \mathbf{H}$ & ${ }^{13} \mathrm{C}$ \\
\hline $\mathbf{a}$ & & & & & & \\
\hline $\mathrm{D}$ & $4.29( \pm 0.02)$ & $61.98( \pm 0.29)$ & $4.24( \pm 0.01)$ & $62.17( \pm 0.07)$ & $4.27( \pm 0.01)$ & $62.06( \pm 0.26)$ \\
\hline $\mathrm{L}(1,2)$ & $4.26( \pm 0.01)$ & $62.57( \pm 0.09)$ & $4.21( \pm 0.01)$ & $62.68( \pm 0.11)$ & $4.24( \pm 0.02)$ & $62.62( \pm 0.19)$ \\
\hline $\mathrm{T}(1,3)$ & $3.44( \pm 0.29)$ & $59.98( \pm 0.05)$ & $3.47( \pm 0.04)$ & $59.85( \pm 0.09)$ & $3.46( \pm 0.03)$ & $59.93( \pm 0.05)$ \\
\hline $\mathrm{L}(1,3)$ & $4.11(+0.08)$ & $65.05(+0.08)$ & $4.07(+0.01)$ & $65.32(+0.08)$ & $4.12(+0.03)$ & $64.88( \pm 0.04)$ \\
\hline TG & $4.08( \pm 0.06)$ & $65.75( \pm 0.11)$ & $4.04( \pm 0.01)$ & $66.00( \pm 0.07)$ & $4.08( \pm 0.02)$ & $65.63( \pm 0.03)$ \\
\hline b & & & & & & \\
\hline $\mathrm{D}$ & $5.22( \pm 0.02)$ & $68.95( \pm 0.26)$ & $5.19( \pm 0.01)$ & $69.09( \pm 0.10)$ & $5.22( \pm 0.04)$ & $68.89( \pm 0.03)$ \\
\hline $\mathrm{L}(1,2)$ & $4.97( \pm 0.01)$ & $72.30( \pm 0.19)$ & $4.94( \pm 0.01)$ & $72.51( \pm 0.15)$ & $4.95( \pm 0.08)$ & $72.29( \pm 0.32)$ \\
\hline $\mathrm{T}(1,3)$ & $4.77( \pm 0.02)$ & $75.61( \pm 0.40)$ & $4.73( \pm 0.02)$ & $75.90( \pm 0.20)$ & $4.75( \pm 0.05)$ & $75.51( \pm 0.03)$ \\
\hline $\mathrm{L}(1,3)$ & $3.90( \pm 0.02)$ & $66.34( \pm 0.11)$ & $3.88( \pm 0.02)$ & $66.26( \pm 0.10)$ & $3.90( \pm 0.04)$ & $66.30( \pm 0.05)$ \\
\hline TG & $3.65( \pm 0.02)$ & $69.59( \pm 0.31)$ & $3.65( \pm 0.01)$ & $69.44( \pm 0.11)$ & $3.66( \pm 0.05)$ & $69.45( \pm 0.04)$ \\
\hline & & & & & & \\
\hline $\mathrm{D}$ & $4.29( \pm 0.02)$ & $62.06( \pm 0.36)$ & $4.24( \pm 0.01)$ & $62.17( \pm 0.07)$ & $4.27( \pm 0.01)$ & $62.06( \pm 0.26)$ \\
\hline $\mathrm{L}(1,2)$ & $3.70( \pm 0.38)$ & $60.48( \pm 1.15)$ & $3.51( \pm 0.02)$ & $59.56( \pm 0.09)$ & $3.50( \pm 0.02)$ & $59.68( \pm 0.04)$ \\
\hline $\mathrm{T}(1,3)$ & $3.45( \pm 0.04)$ & $59.89( \pm 0.16)$ & $3.47( \pm 0.04)$ & $59.85( \pm 0.09)$ & $3.46( \pm 0.03)$ & $59.93( \pm 0.05)$ \\
\hline $\mathrm{L}(1,3)$ & $3.93( \pm 0.33)$ & $63.63( \pm 2.44)$ & $4.07( \pm 0.01)$ & $65.33( \pm 0.08)$ & $4.12( \pm 0.03)$ & $64.88( \pm 0.04)$ \\
\hline TG & $3.52( \pm 0.35)$ & $63.43( \pm 0.95)$ & $3.39( \pm 0.03)$ & $63.23( \pm 0.07)$ & $3.39( \pm 0.03)$ & $63.11( \pm 0.22)$ \\
\hline
\end{tabular}

The ${ }^{13} \mathrm{C}$ NMR spectra of most of the dilute-solution samples revealed the presence of two clearly distinguishable peaks associated with the central glycerol carbon (Cb) for linear polymerization (especially $\mathrm{L}_{1,2}$, but also to a lesser extent $\mathrm{L}_{1,3}$ and $\mathrm{T}_{\mathrm{G}}$, see supplementary material). The only samples in which this was not observed were the two neat samples (Table 2, lines 13 and 15), the $72 \mathrm{~h}$ azelaic:glycerol (2:1 molar ratio) polymer (Table 2, line 11) and the glutaric:glycerol (2:1 molar ratio) polymer made at $135{ }^{\circ} \mathrm{C}$ (Table 2, line 8). These two peaks were most frequently observed in those samples which had medium-to-low dendritic branching (D.B. $<\sim 33 \%$ ). The relative intensities of these peaks vary amongst the samples and may be somewhat dependent upon preparation method, although no clear pattern could be discerned. The most likely explanation is that these samples consist of a mixture of long and short-chain linear polymers. It was also observed that some samples had multiple ${ }^{13} \mathrm{C}$ peaks associated with dendritic branching. These peaks were different from the $\mathrm{L}_{1,2}$ peaks just mentioned in that in most cases they appear as a splitting of the main " $D$ " peak and are not well resolved. This was observed in all of the glutaric acid:glycerol (2:1 molar ratio) products and the succinic acid:glycerol (2:1 molar ratio) polymer prepared at $135{ }^{\circ} \mathrm{C}$ (Table 1 , line 6). All of these samples have higher levels of dendritic branching (> 20\%). While these might also arise from a mixture of long and short-chains, their presence in the neat samples suggests that they arise from 
structures that are more likely to be found in higher concentrations. Hence we postulate that these arise from cyclic polymer structures, which are not possible in structures consisting of linear branching.

Table 2. (a) Relative amounts of glycerol branching (D.B.\%) in oligo(glycerol-diacid)s calculated from ${ }^{1} \mathrm{H}$ NMR spectral data for oligo(glycerol-diacids) using Equation (1) ${ }^{\mathrm{a}, \mathrm{b}, \mathrm{c}}$. (b) Relative amounts of glycerol branching (D.B.\%) for oligo(glycerol-diacid)s calculated from ${ }^{13}$ C NMR spectral data for oligo(glycerol-diacids) using Equation (1) ${ }^{\text {a,b,c }}$.

\begin{tabular}{|c|c|c|c|c|c|c|c|}
\hline & Diacid & $\mathbf{D}$ & $\mathbf{L}(\mathbf{1 , 2})$ & $\mathbf{T}(1,3)$ & $\mathbf{L}(1,3)$ & $\mathbf{T}_{\mathbf{G}}$ & D.B.\% \\
\hline \multicolumn{8}{|c|}{ 1:1 molar ratio to glycerol (no Dean Stark apparatus), $110{ }^{\circ} \mathrm{C}, 24 \mathrm{~h}$} \\
\hline 1 & Succinic & 3.5 & 9.7 & 7.3 & 33.7 & 45.9 & 13.7 \\
\hline 2 & Glutaric & 5.2 & 11.1 & 4.4 & 44.8 & 34.5 & 15.7 \\
\hline 3 & Azelaic & 4.1 & 12.6 & 4.3 & 42.6 & 36.4 & 13.0 \\
\hline \multicolumn{8}{|c|}{ 1:1 molar ratio to glycerol, $135^{\circ} \mathrm{C}, 24 \mathrm{~h}$} \\
\hline 4 & Succinic & 13.74 & 22.7 & 8.51 & 35.8 & 19.3 & 31.8 \\
\hline 5 & Glutaric & \multicolumn{6}{|c|}{ (Peaks too broad to interpret-see text) } \\
\hline 6 & Azelaic & 2.6 & 12 & 4.8 & 33.3 & 47.4 & 10.2 \\
\hline \multicolumn{8}{|c|}{ 2:1 molar ratio to glycerol, $135^{\circ} \mathrm{C}, 24 \mathrm{~h}$} \\
\hline 7 & Succinic & 22.0 & 25.6 & 8.88 & 34.4 & 9.08 & 42.4 \\
\hline 8 & Glutaric & 61.3 & 8.3 & 1.2 & 18.2 & 11 & 82.2 \\
\hline 9 & Azelaic & 3.2 & 17.9 & 4.78 & 21.0 & 53.1 & 14.1 \\
\hline \multicolumn{8}{|c|}{ 1:1 molar ratio to glycerol, $155^{\circ} \mathrm{C}, 24 \mathrm{~h}$} \\
\hline \multirow{2}{*}{\multicolumn{8}{|c|}{$\begin{array}{r}\text { (Peaks too broad to interpret-see text) } \\
2: 1 \text { molar ratio with glycerol, } 135^{\circ} \mathrm{C}, 72 \mathbf{h}\end{array}$}} \\
\hline & & & & & & & \\
\hline 11 & Azelaic & 24.7 & 40.7 & 1.2 & 28.4 & 5.1 & 41.7 \\
\hline \multicolumn{8}{|c|}{ 2:1 molar ratio with glycerol, $135^{\circ} \mathrm{C}, 10 \mathrm{~h}$} \\
\hline 12 & Glutaric & 47.5 & 18.6 & .5 & 29.1 & 4.3 & 66.6 \\
\hline \multicolumn{8}{|c|}{ 2:1 molar ratio with glycerol, $155^{\circ} \mathrm{C}$, neat, $10 \mathrm{~h}$} \\
\hline 13 & Glutaric & 52.6 & 15.0 & 3.6 & 24.3 & 4.4 & 72.9 \\
\hline \multicolumn{8}{|c|}{$2: 1$ molar ratio to glycerol, $155^{\circ} \mathrm{C}, 24 \mathrm{~h}$} \\
\hline 14 & Glutaric & & (Peak & broad & terpret - & text) & \\
\hline \multicolumn{8}{|c|}{ 2:1 molar ratio with glycerol, $155^{\circ} \mathrm{C}$, neat, $24 \mathrm{~h}$} \\
\hline 15 & Glutaric & & $(\mathrm{Pea}$ & o broad & nterpret- & text) & \\
\hline
\end{tabular}

(a)

\begin{tabular}{|c|c|c|c|c|c|c|c|}
\hline & Diacid & D & $\mathbf{L}(\mathbf{1}, \mathbf{2})$ & $\mathbf{T}(1,3)$ & $\mathbf{L}(1,3)$ & $\mathbf{T}_{\mathbf{G}}$ & D.B.\% \\
\hline \multicolumn{8}{|c|}{ 1:1 molar ratio to glycerol (no Dean Stark apparatus), $110{ }^{\circ} \mathrm{C}, 24 \mathrm{~h}$} \\
\hline 1 & Succinic & 3.5 & 30.7 & 3.5 & 22.9 & 43.0 & 11.5 \\
\hline 2 & Glutaric & 4.1 & 29.7 & 4.6 & 24.6 & 37.0 & 13.2 \\
\hline 3 & Azelaic & 4.4 & 28.4 & 5.1 & 21.8 & 40.3 & 15.0 \\
\hline \multicolumn{8}{|c|}{ 1:1 molar ratio to glycerol, $135^{\circ} \mathrm{C}, 24 \mathrm{~h}$} \\
\hline 4 & Succinic & 7.8 & 23.9 & 5.1 & 30.2 & 33.0 & 39.4 \\
\hline 5 & Glutaric & 13.7 & 19.6 & 3.0 & 40.7 & 23.1 & 31.2 \\
\hline \multirow[t]{2}{*}{6} & Azelaic & 2.8 & 32.5 & 5.5 & 18.8 & 40.4 & 9.9 \\
\hline & Diacid & $\mathbf{D}$ & $\mathbf{L}(\mathbf{1}, \mathbf{2})$ & $\mathbf{T}(1,3)$ & $\mathbf{L}(\mathbf{1 , 3 )}$ & $\mathbf{T}_{\mathrm{G}}$ & D.B.\% \\
\hline
\end{tabular}


Table 2. Cont.

\begin{tabular}{|c|c|c|c|c|c|c|c|}
\hline \multicolumn{8}{|c|}{ 2:1 molar ratio to glycerol, $135^{\circ} \mathrm{C}, 24 \mathrm{~h}$} \\
\hline 7 & Succinic & 9.1 & 19.4 & 3.0 & 37.6 & 31.0 & 41.9 \\
\hline 8 & Glutaric & 63.9 & 5.6 & 0 & 15.9 & 14.5 & 85.6 \\
\hline 9 & Azelaic & 4.0 & 27.0 & 4.9 & 22.1 & 42.1 & 13.9 \\
\hline \multicolumn{8}{|c|}{ 1:1 molar ratio to glycerol, $155^{\circ} \mathrm{C}, 24 \mathrm{~h}$} \\
\hline 10 & Glutaric & 15.6 & 19.5 & 0.9 & 43.2 & 20.8 & 33.3 \\
\hline \multicolumn{8}{|c|}{ 2:1 molar ratio with glycerol, $135^{\circ} \mathrm{C}, 72 \mathrm{~h}$} \\
\hline 11 & Azelaic & 27.4 & 16.2 & 1.2 & 41.9 & 13.4 & 48.5 \\
\hline \multicolumn{8}{|c|}{ 2:1 molar ratio with glycerol, $135^{\circ} \mathrm{C}, 10 \mathrm{~h}$} \\
\hline 12 & Glutaric & 54.7 & 5.0 & 0.0 & 39.8 & 0.5 & 70.9 \\
\hline \multicolumn{8}{|c|}{ 2:1 molar ratio with glycerol, $155^{\circ} \mathrm{C}$, neat, $10 \mathrm{~h}$} \\
\hline 13 & Glutaric & 50.8 & 11.8 & 0.0 & 30.7 & 6.7 & 70.5 \\
\hline \multicolumn{8}{|c|}{ 2:1 molar ratio with glycerol, $155^{\circ} \mathrm{C}, 24 \mathrm{~h}$} \\
\hline 14 & Glutaric & 62.2 & 11.5 & 0.0 & 26.3 & 0.0 & 76.7 \\
\hline \multicolumn{8}{|c|}{ 2:1 molar ratio with glycerol, $155^{\circ} \mathrm{C}$, neat, $24 \mathrm{~h}$} \\
\hline 15 & Glutaric & 78.2 & 3.9 & 0 & 17.9 & 0.0 & 87.8 \\
\hline
\end{tabular}

(b)

n.d. = not determined. ${ }^{\text {a }}$ All experiments are performed for $24 \mathrm{~h}$ and in toluene unless otherwise noted;

${ }^{\mathrm{b}}$ All experiment are ran using a Dean Stark apparatus, except data in lines 1,2,3,13, and 15; ${ }^{\mathrm{c}}$ Rounding errors may not result in exactly 100\%; D.B.\% calculation performed on raw numbers.

\subsection{Degree of Branching (D.B.\%)}

The assignments and integrations of both the ${ }^{13} \mathrm{C}$ and ${ }^{1} \mathrm{H}$ spectra (Table 1 ) were used for the calculation and comparison of their respective degree of branching ratios (Table 2(a,b)). As previously mentioned, the ${ }^{13} \mathrm{C}$ integrations in this study are considered to be more accurate and reliable than the ${ }^{1} \mathrm{H}$ values. Therefore, while the D.B.\% values obtained from the assignment and integration of the ${ }^{1} \mathrm{H}$ spectra are available for comparison in Table 2(a), the ${ }^{13} \mathrm{C}$ data in Table 2(b) will be the primary focus of the following discussion.

General observations of the degree of branching results reveal that the 2:1 (diacid:glycerol) formulations gave products with the highest D.B.\%. The polymers co-polymerized with glutaric acid at temperatures at or above $135{ }^{\circ} \mathrm{C}$ produced materials with D.B.\% that ranged from $70.5 \%$ for a $10 \mathrm{~h}$ reaction to $87.8 \%$ for a $24 \mathrm{~h}$ reaction. Under similar conditions, the analoguous products made from succinic acid and azelaic acid gave considerably lower degrees of branching (Table 2, lines 7 and 9). Even increasing the reaction time for the synthesis of the poly(glycerol-azelaic acid) to $72 \mathrm{~h}$ (Table 2, line 11$)$ only gave a product with a D.B.\% of $48.5 \%$. Allowing the neat, glutaric acid:glycerol (2:1) reaction to proceed for $24 \mathrm{~h}$, yielded a clear, colorless, flexible material with a high degree of branching (Table 2, line 15) rather than a thick viscous gel as observed for the complimentary reaction performed in toluene which resulted in a lower degree of branching (Table 2, line 14). Despite this observation, there seems to be no correlation between D.B.\% and reaction medium. In fact, when comparing the glutaric-acid derived polymers, temperature seems to play a role in how much the polymers branch and, surprisingly, it appears that product synthesized in toluene at $135{ }^{\circ} \mathrm{C}$ (Table 2, 
line 8) branch to a higher degree than those synthesized at $155^{\circ} \mathrm{C}$ (Table 2, line 14). Even when a neat reaction was performed at $155^{\circ} \mathrm{C}$ for $10 \mathrm{~h}$ (Table 2 line 13), the D.B.\% was comparable to that of a reaction performed in toluene at $135{ }^{\circ} \mathrm{C}$ (Table 2, line 12) for the same amount of time. It may be of interest to further investigate a larger set of diacids to determine if this observation for the glutaric acid-derived polymer is a unique occurrence or if its chemical or physical properties, such as solubility, polarity, and the spatial arrangement of its atoms, play a role in increasing its degree of branching. When using 1:1 molar ratios of glycerol and diacid, polymers with D.B.\% lower than $40 \%$ resulted even when reacted for $24 \mathrm{~h}$ at $155^{\circ} \mathrm{C}$ (Table 2, lines $4-6,10$ ). For the 1:1 formulation, the difference in D.B.\% for the succinic acid and glutaric acid-derived materials may not be significant. However, as observed for the 2:1 forumation, the azelaic acid derived polymer continues to branch less frequently than the succinic acid and glutaric acid analogues. While keeping all other reaction conditions the same for the esterification of glycerol with glutaric acid (1:1 molar ratio), increasing the oil bath temperature from $135{ }^{\circ} \mathrm{C}$ to $155{ }^{\circ} \mathrm{C}$ resulted in crosslinked hydrogels that were solid and insoluble in all solvents. However, the degree of branching only increased by $2.1 \%$ (Table 2, lines 5 and 10) suggesting that the degree of branching here represents intermolecularly crosslinked polymers that are more energetically favored at higher temperatures and can therefore be controlled. It was previously observed and discussed in reference 16 that water was not transferred into the Dean-Stark apparatus unless the oil bath temperature was heated to at least $135{ }^{\circ} \mathrm{C}$. Therefore, performing the reaction at any temperature lower than $135{ }^{\circ} \mathrm{C}$ was the equivalent to performing the reaction without the Dean-Stark apparatus. Removing the Dean-Stark apparatus resulted in a $\sim 47 \%$ decrease in degree of branching for both the succinic acid-derived polymers and the glutaric acid-derived polymers, indicating either that hydrolysis of the ester bond was faster than esterification of the secondary alcohol on glycerol or that the energy required to esterify the secondary alcohol on the glycerol was not sufficient at reflux.

\section{Conclusions}

${ }^{1} \mathrm{H}$ NMR and ${ }^{13} \mathrm{C}$ NMR (1-dimensional and 2-dimensional) has been used to successfully assign and integrate chemical resonances and determine the degrees of branching along the glycerol backbone of polyesters synthesized from glycerol and diacids in the presence and absence of toluene (solvent). However, there is no indication that degree of branching is significantly affected by the presence or absence of solvent according to the trends observed in this research. Glutaric acid-derived polymers 2:1 (diacid:glycerol) gave the highest degree of polymer branching after the $24 \mathrm{~h}$ reaction period followed by the 2:1 formulations for succinic acid-derived polymers and the azelaic acid-derived polymers, respectively. In general, polymers made from a 1:1 molar ratio of glycerol and diacid gave less than $40 \%$ branching. Reactions performed at reflux for $24 \mathrm{~h}$ resulted in a $70.8 \%$ and $56.7 \%$ decrease in degree of branching for succinic acid and glutaric acid-derived polyesters, respectively, when compared to the analogous reactions using a Dean-Stark apparatus performed in a $135{ }^{\circ} \mathrm{C}$ oil bath.

\section{Acknowledgments}

The contributions from Aisha Abdul-Wakeel are acknowledged for her assistance with the collection and analysis of NMR data. 


\section{Disclaimer}

Mention of trade names or commercial products in this paper is solely for the purpose of providing specific information and does not imply recommendation or endorsement by the U.S. Department of Agriculture.

\section{References}

1. Lin, Q.; Long, T.E. Polymerization of $\mathrm{A}_{2}$ with $\mathrm{B}_{3}$ monomers: A facile approach to hyperbranched poly(aryl ester)s. Macromolecules 2003, 36, 9809-9816.

2. Stumbe, J.-F.; Bruchmann, B. Hyperbranched polyesters based on adipic acid and glycerol Macromol. Rapid Commun. 2004, 25, 921-924.

3. Kulshrestha, A.S.; Gao, W.; Gross, R.A. Glycerol copolyesters: Control of branching and molecular weight using lipase catalyst. Macromolecules 2005, 38, 3193-3204.

4. Jikei, M.; Kakimoto, M.A. Hyperbranched aromatic polyamides prepared by direct polycondensation. High Perform. Polym. 2001, 13, 33-43.

5. Fang, J.; Kita, H.; Okamoto, K. Hyperbranched polyimide for gas separation applications. 1. Synthesis and characterization. Macromolecules 2000, 33, 4639-4646.

6. Komber, H.; Voit, B.; Monticelli, O.; Russo, S. ${ }^{1} \mathrm{H}$ and ${ }^{13} \mathrm{C}$ Spectra of a hyperbranched aromatic polyamide from p-phenylenediamine and trimesic acid. Macromolecules 2001, 34, 5487-5493.

7. Wyatt, V.T.; Haas, M.J.; Strahan, G.D.; Nunez, A. Characterization of thermal and mechanical properties of hyperbranched oligo(glycerol-glutaric acid)s. J. Biobased Mater. Bioenergy 2011, 5, 92-101.

8. Voit, B.I. Dendritic polymers: From aesthetic macromolecules to commercially interesting materials. Acta Polym. 1995, 46, 87-99.

9. Tomalia, D.A.; Frechet, J.M.J. Discovery of dendrimers and dendritic polymers: A brief historical perspective. J. Polym. Sci. Part A: Polym. Chem. 2002, 40, 2719-2728.

10. Kim, Y.H. Hyperbranched polymers 10 years after. J. Polym. Sci. Part A: Polym. Chem. 1998, 36, 1685-1698.

11. Haag, R.; Sunder, A.; Stumbe, J.-F. An approach to glycerol dendrimers and pseudo-dendritic polyglycerols. J. Am. Chem. Soc. 2000, 122, 2954-2955.

12. Sunder, A.; Hanselmann, R.; Frey, H.; Mulhaupt, R. Controlled synthesis of hyperbranched polyglycerols by ring-opening multibranching polymerization. Macromolecules 1999, 32, 4240-4246.

13. Holter, D.; Burgath, A.; Frey, H. Degree of branching in hyperbranched polymers. Acta Polym. 1997, 48, 30-35.

14. Holter, D.; Frey, H. Degree of branching in hyperbranched polymers. 2. Enhancement of DB: Scope and limitations. Acta Polym. 1997, 48, 298-309.

15. Flory, P.J. Molecular size distribution in three dimensional polymers. I. Gelation. J. Am. Chem. Soc. 1941, 63, 3083-3096.

16. Wyatt, V.T. The Lewis-Acid catalyzed synthesis of hyperbranched polymers based on glycerol and diacids in toluene. J. Am. Oil Chem. Soc. 2012, 89, 313-319. 
17. Wyatt, V.T.; Nuñez, A.; Strahan, G.D. The Lewis acid-catalyzed synthesis of hyperbranched oligo(glycerol-diacid)s in aprotic polar media. J. Am. Oil Chem. Soc. 2010, 87, 1539-1369.

18. Frazza, E.J.; Schmitt, E.E. A new absorbable suture. J. Biomed. Mater. Res. Symp. 1971, 1, 43-58.

19. Vert, M.; Li, S.M. Bioresorbability and biocompatibility of aliphatic polyesters J. Mater. Sci. Mater. Med. 1992, 3, 432-446.

20. Shalaby, S.W.; Johnson, R.A. Biomedical Polymers; Shalaby, S.W., Ed.; Hanser Publishing: New York, NY, USA, 1994; Chapter 1, pp. 2-34.

21. Seiler, M.; Kohler, D.; Arlt, W. Hyperbranched polymers: New selective solvents for extractive distillation and solvent extraction. Sep. Purif. Technol. 2003, 30, 179-197.

22. Rabiller, C.; Maze, F. Quantitative analysis and determination of the enantiomeric purity of glycerides by ${ }^{13} \mathrm{C}$ NMR Spectroscopy. Application to the lipase-catalysed transesterification of triacylglycerides. Magn. Reson. Chem. 1989, 27, 582-584.

23. Halldorsson, A.; Magnusson, C.D.; Haraldsson, G.G. Chemoenzymatic synthesis of structured triacylglycerols by highly regioselective acylation. Tetrahedron 2003, 59, 9101-9109.

24. Magnusson, H.;Malmstrum, E.; Hult, A. Influence of reaction conditions on degree of branching in byperbranced aliphatic polyethers from 3-ethyl-3 (hydroxymethyl)oxetane. Macromolecules 2001, 34, 5786-5791.

25. Hawker, C.J.; Lee, R.; Frechet, J.M.J. One-step synthesis of hyperbranched dendritic polyesters. J. Am. Chem. Soc. 1991, 113, 4583-4588.

26. Kim, Y.H. Highly branched aromatic polymers prepared by single step syntheses. Macromol. Symp. 1994, 77, 21-33.

(C) 2012 by the authors; licensee MDPI, Basel, Switzerland. This article is an open access article distributed under the terms and conditions of the Creative Commons Attribution license (http://creativecommons.org/licenses/by/3.0/). 